\title{
Clinical value of soluble ST2 in cardiology
}

\author{
Magdalena Dudek ${ }^{A, B, D, F}$, Marta Kałużna-Oleksy ${ }^{B, D}$, Jacek Migaj ${ }^{B, D}$, Ewa Straburzyńska-Migaj ${ }^{\mathrm{A}, D-F}$ \\ ${ }^{\text {st }}$ Department of Cardiology, Poznan University of Medical Sciences, Poland \\ A - research concept and design; B - collection and/or assembly of data; $C$ - data analysis and interpretation; \\ $D$ - writing the article; $E$ - critical revision of the article; $F$ - final approval of the article
}

\section{Address for correspondence \\ Magdalena Dudek}

E-mail: magdalena.dudek@skpp.edu.pl

Funding sources

None declared

\section{Conflict of interest}

None declared

Received on April 27, 2020

Reviewed on June 28,2020

Accepted on August 5, 2020

Published online on October 13, 2020

\begin{abstract}
We are constantly looking for new parameters and markers that can help in the assessment of patients with various diseases, including cardiac disorders; this can translate into better care and improved prognosis. Suppression of tumorigenicity 2 (ST2) has recently gained interest as a potential biomarker in many fields: it is involved in many inflammatory diseases and allergies, including asthma, rheumatoid arthritis and inflammatory bowel disease, and it participates in cardiovascular pathophysiology. Suppression of tumorigenicity 2 is being investigated as a promising biomarker in heart diseases. The interaction of interleukin 33 (IL-33) and ST2L is part of a cardioprotective pathway that prevents fibrosis and inhibits inflammatory response, hypertrophy and apoptosis of cardiomyocytes. In this review, we try to summarize the current knowledge about the usefulness of soluble ST2 (sST2) in cardiology. Clinical data show promising results for the possibility of using SST2 in various diseases, such as arrhythmia, hypertension, myocarditis, acute aortic syndrome, and coronary artery disease (CAD). This novel biomarker may also play a role in heart transplantation and perioperative care.
\end{abstract}

Key words: heart, biomarker, ST2, cardiac biomarkers, SST2

Cite as

Dudek M, Kałużna-Oleksy M, Migaj J, Straburzyńska-Migaj E. Clinical value of soluble ST2 in cardiology. Adv Clin Exp Med. 2020;29(10):1205-1210. doi:10.17219/acem/126049

D0I

10.17219/acem/126049

\section{Copyright}

Copyright by Author(s)

This is an article distributed under the terms of the

Creative Commons Attribution 3.0 Unported (CC BY 3.0)

(https://creativecommons.org/licenses/by/3.0/) 


\section{Introduction: sST2}

\section{as a new biomarker}

In 2001, the Biomarkers Definitions Working Group, a part of the National Institutes of Health (NIH, Bethesda, USA), has updated the definition of a biomarker as "a characteristic that is objectively measured and evaluated as an indicator of normal biologic processes, pathogenic processes or pharmacologic responses to a therapeutic intervention". ${ }^{1}$ New parameters and markers that can help improve the assessment of patients with various diseases, including cardiac disorders, are constantly being sought; they can translate into better care and improved prognosis. Suppression of tumorigenicity 2 (ST2) has recently gained interest as a potential biomarker in many fields. A member of the interleukin 1 (IL-1) receptor/Toll-like superfamily, ST2 is known as interleukin 1 receptor-like 1 (IL1RL-1) and when it was originally described in 1989, it was considered an "orphan" receptor. ${ }^{2}$ Interleukin 33 (IL-33), a member of the IL-1 family of cytokines, was described as a ligand for the suppression of tumorigenicity 2 in $2005 .^{3}$ The discovery of IL-33 helped to understand the signaling axis of IL-33 and ST2. The ST2L, after IL-33 binding, has an inhibitory effect on the inflammatory response related to Th2 (Fig. 1).

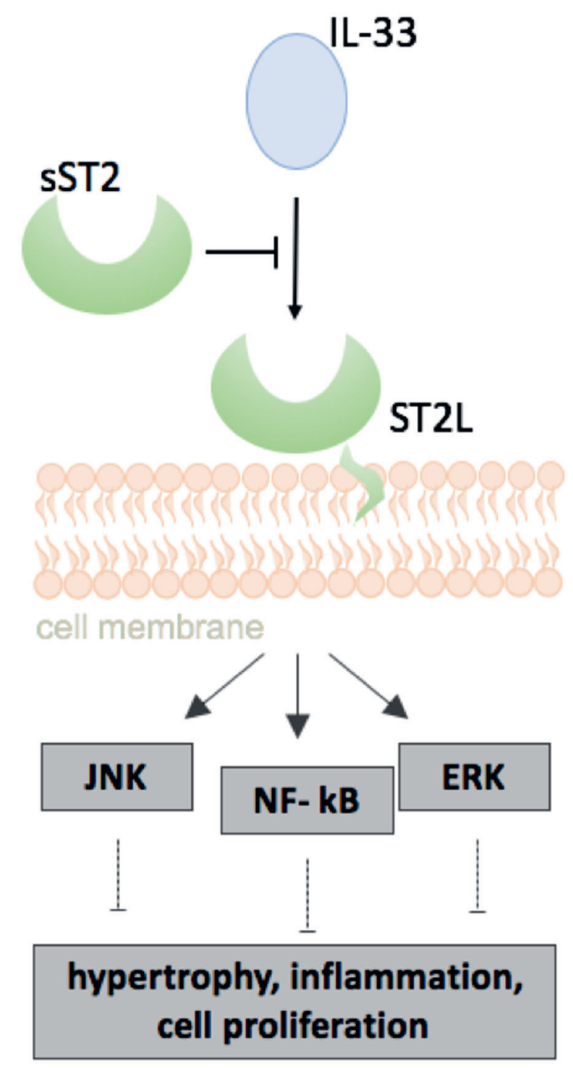

Fig. 1. Signaling axis of IL-33 and ST2

IL-33 - interleukin 33; sST2 - soluble suppression of tumorigenesis 2; ST2L - membrane-bound receptor; JNK - c-Jun N-terminal kinases; NF-kB - nuclear factor kappa-light-chain-enhancer of activated B cells; ERK - extracellular signal-regulated kinases.
Suppression of tumorigenicity 2 participates in inflammatory processes and functions in relation to immune diseases. To the best of our current knowledge, ST2 has 4 isoforms: the 2 main ones are a membrane-bound receptor form (ST2L) and a soluble form (sST2), while the other 2 are ST2V ${ }^{4}$ and ST2LV. ${ }^{5}$ Differences in their structure and quantity result from a dual promoter system to drive differential mRNA expression. ${ }^{6}$ The ST2L membrane protein consists of 3 extracellular domains, a single transmembrane domain and an intracellular domain. ${ }^{6}$ Because sST2 lacks the transmembrane and intracellular domains, it circulates freely in the blood. Interleukin 33, due to tissue damage or necrosis, is released into the extracellular space, where it binds to the ST2 receptor and then recruits the IL-1 receptor accessory protein (IL-1RAcP), which leads to the activation of the NK-kB signaling pathway. ${ }^{7}$ The soluble form of ST2, after binding to IL33, inhibits the inflammatory response associated with Th2 lymphocytes, thereby blocking the protective effect of IL-33 (Fig. 1).

Interleukin 33 is biologically active and may be released by living and necrotic cells, the latter as a result of tissue damage playing the role of an endogenous danger signal. ${ }^{8}$ Multiple organs and cell types in humans express IL-33. ${ }^{3}$ Together with ST2, IL-33 is involved in many inflammatory and allergic diseases, including asthma, ${ }^{9}$ rheumatoid arthritis ${ }^{10}$ and inflammatory bowel disease, ${ }^{11}$ and it participates in cardiovascular pathophysiology. Weinberg et al. described the expression of ST2 in cardiac cells as a "response" to myocardial stress and biomechanical overload. ${ }^{12}$ This discovery focused researchers' attention on the role of ST2 in the cardiovascular system. Further studies revealed that in cardiac diseases, the main source of sST2 may be vascular endothelial cells rather than myocardium. ${ }^{13}$ The interaction of IL-33 and ST2L is part of the cardioprotective pathway that prevents fibrosis and inhibits the inflammatory response, hypertrophy, and apoptosis of cardiomyocytes.

\section{Soluble ST2 and coronary artery disease}

Circulating ST2 is a promising biomarker being investigated in ischemic heart disease. Several studies have investigated the association of sST2 serum levels with the prognosis of coronary artery disease (CAD). Myocardial infarction (MI) with ST-segment elevation (STEMI) is associated with increased myocardial expression of sST2 and elevated levels of serum sST2 due to transmural myocardial injury and stretching of the left ventricle. ${ }^{14}$ During this process, cardiac fibroblasts and cardiomyocytes release IL-33 and sST2. ${ }^{14}$ In 2002, Weinberg et al. showed that sST2 was elevated after experimental MI in mice. A similar situation was observed in vivo, in patients 1 day after MI. ${ }^{12}$ Several studies have reported that an increased sST2 level in the initial phase of STEMI is a significant 
prognostic biomarker for predicting mortality and heart failure (HF). ${ }^{15,16}$ Using this assay can help identify patients with a higher risk of developing adverse cardiac events while in intensive care due to MI. ${ }^{16}$ O'Donoghue et al. showed that sST2 level in patients hospitalized with acute MI is a significant predictor of short-term cardiovascular death or the development of $\mathrm{HF}^{17}$ A high concentration of sST2 is an important predictor of major adverse cardiovascular events (MACE), including cardiovascular mortality and HF in a one-year follow-up period in MI patients. ${ }^{18}$ Moreover, sST2 may be used as a predictor of late ventricular remodeling after MI. ${ }^{19}$ Although baseline sST2 level may be valuable in predicting MACEs in STEMI patients receiving primary $\mathrm{PCI},{ }^{20}$ there are no guidelines that recommend $\mathrm{SST} 2$ testing as a predictor biomarker in STEMI patients.

The concentration of SST2 may be used to predict cardiovascular risk. An increased level of this biomarker was an independent predictor of long-term all-cause mortality for stable CAD. ${ }^{21}$ Zhang et al., in a recently published study, confirmed that sST2 concentration was not related to the severity of coronary artery atherosclerosis detected with angiography. ${ }^{22}$ However, they found an association between complex coronary lesion morphology and sST2 level, especially in a group of patients with unstable CAD. The authors suggested that SST 2 concentration may be useful as a marker in the detection of unstable and complex atherosclerotic plaques. $^{22}$

\section{Soluble ST2 and acute aortic syndrome}

Another group of diseases in which the sST2 biomarker can be used is acute aortic syndromes (AAS). Wang et al. showed that AAS might result in the release and elevation of serum sST2 concentration. ${ }^{23}$ Moreover, they indicated the potential superiority of sST2 over D-dimer in AAS. Morello et al. confirmed higher levels of serum sST2 in patients with AAS, but, unlike previous studies, showed poor diagnostic utility. ${ }^{24}$ Further studies are needed in this field to confirm the usefulness SST2 as a biomarker in aortic diseases.

\section{Soluble ST2 and myocarditis}

Soluble ST2 may also be used as a biomarker in patients with myocarditis for predicting the development of HF. In a recent study, Coronado et al. showed higher sST2 concentrations in both men and women with myocarditis, ${ }^{25}$ but sST2 level only correlated with worse HF symptoms based on New York Heart Association Functional Classification (NYHA) class in men $\leq 50$ years old. This was the first work to show the potential usefulness of sST2 in myocarditis; more studies in this field are required.

\section{Soluble ST2 and arterial hypertension}

Soluble ST2 may be useful as a diagnostic biomarker for cardiac remodeling in patients with arterial hypertension (AH). It has been shown that plasma concentration of sST2 in the general population is associated with systolic blood pressure (SBP). ${ }^{26}$ Hypertensive patients with HF and left ventricular hypertrophy (LVH) had higher plasma sST2 concentrations than those without LVH. This biomarker has the potential to distinguish AH patients with or without $\mathrm{LVH} .{ }^{27}$ Similar findings were reported in a study by Ojji et al., where sST2 may help differentiate patients with LVH in the HA population. ${ }^{27}$ Farcas et al. confirmed that in hypertensive patients' serum, sST2 levels increase along with LV mass and the severity of myocardial dysfunction. ${ }^{28}$ Moreover, sST2 level can help in predicting LV diastolic dysfunction in hypertensive patients, an indicator which displayed a significant positive correlation with impaired relaxation parameters (transmitral E/A ratio) measured echoardiographically. ${ }^{28}$

\section{Soluble ST2 and atrial fibrillation}

In the most common clinically relevant arrhythmia - atrial fibrillation (AF) - sST2 is still under investigation. Chen et al. showed that sST2 levels were higher in patients with AF, both persistent and paroxysmal, than in patients with sinus rhythm. ${ }^{29}$ In patients with CAD, serum levels of sST2 predict the risk of new-onset AF. ${ }^{30}$ Okar et al. demonstrated the possible utility of sST2 as a new biomarker to predict the reoccurrence of paroxysmal AF in patients after cryoballoon catheter ablation. ${ }^{31}$ Soluble ST2 may be applied as an independent biomarker for predicting $\mathrm{HF}$ in patients with AF. ${ }^{29}$ The CASABLANCA study showed that sST2 adds an independent prognostic value in the population at a high risk of HF for predicting progression to the development of symptomatic HF. ${ }^{32}$ The role of soluble ST2 in AF still has to be precisely explored; additional studies should be conducted to validate these findings.

\section{Soluble ST2 and heart failure}

Soluble ST2 is a biomarker for the prognosis of acute and chronic HF, and may be used for predicting all-cause and cardiac mortality among HF patients, and even in the general population. Elevated concentrations of sST2 are associated with poorer LVF and higher NYHA functional class. According to some studies, age, sex, body mass index (BMI), etiology of HF, and comorbidities such as renal dysfunction has less of an influence on SST2 levels than on natriuretic peptides. ${ }^{33}$ Soluble ST2 is considered a valuable biomarker used in the prediction and monitoring of $\mathrm{HF}$ and 
was included in the 2017 American College of Cardiology/ American Heart Association update of HF guidelines. ${ }^{34}$ The first study of sST2 measurement in patients with suspected or proven HF was the PRIDE (Pro-BNP Investigation of Dyspnea in the Emergency Department) substudy. ${ }^{35}$ Soluble ST2 was measured in almost 600 patients with dyspnea with and without HF; the concentration of sST2 was significantly higher in the group of patients with HFrelated dyspnea. ${ }^{35}$ Despite higher levels of sST2 in patients with acute decompensated HF, there was no statistically significant value in the diagnosis of $\mathrm{HF},{ }^{35}$ which was confirmed in further studies. The sST2 levels in the PRIDE study were significantly higher in patients who had died within the one-year follow-up period than in survivors. Moreover, there was an association between sST2 concentration and mortality rates: higher levels predicted a higher risk. Mueller et al. reported similar results that higher sST2 concentration at initial presentation in patients with acute heart failure (AHF) indicated an increased risk of mortality in the future. ${ }^{36}$

After proving that the baseline sST2 values at admission predict outcomes, serial measurements were investigated for its significance. Boisot et al. were the first to note changes in sST2 concentration in hospitalized patients with AHF. ${ }^{37}$ Serial measurements of sST2 correlated with other biomarkers, according to the RELAX-AHF trial, and are useful for prognosis in AHF. ${ }^{38}$ The TRIUMPH study showed that baseline levels and repeated sST2 measurements, performed in patients with AHF, are strong and independent factors of adverse outcome in this group. ${ }^{39}$

Soluble ST2 is gaining attention as a potential tool in the management of chronic heart failure (CHF) and as a prognostic marker in such patients. Concentrations of sST2 in a group of patients with CHF were generally higher than in a healthy population. ${ }^{35}$ Soluble ST2 is an indicator of prognosis in CHF. Patients with higher concentrations of sST2 correlated with worse functional status and more advanced HF. Higher baseline levels of sST2 were associated with worse prognosis and an increased risk of sudden cardiac death in patients with $\mathrm{CHF}{ }^{40,41}$ Soluble ST2 concentration carries information in addition to levels of N-terminal pro-B-type natriuretic peptide (NT-proBNP). Lupón et al. investigated different biomarker combinations to determine their role in CHF. They developed a calculator that takes into account the concentration of sST2 together with NT-pro-BNP and high sensitive troponin $\mathrm{T}$ (hs-TnT) to estimate the risk of death and/or HF hospitalization within 5 years. ${ }^{42}$ The Barcelona Bio-HF Risk Calculator takes into account biomarkers in combination with well-established risk factors such as gender, age, etiology of HF, LVEF, NYHA functional class, estimated glomerular filtration rate (eGFR), diabetes mellitus (DM), sodium and hemoglobin levels, and the treatment administered - $\beta$ a-blocker or angiotensin converting enzyme inhibitor/angiotensin receptor blocker (Table 1). According to meta-analysis of Emdin et al., concentration of sST2
Table 1. Variables used in the Barcelona Bio-Heart Failure Risk Calculator (BCN Bio-HF Calculator)

\begin{tabular}{|l|c|c|}
\hline \multicolumn{1}{|c|}{ Clinical variable } & Treatment & Biomarkers \\
\hline Age & loop diuretics & hs-cTnT \\
\hline Sex & ACE-I or ARB & ST2 \\
\hline NYHA functional class & B-blocker & NT-proBNP \\
\hline LVEF & statin & \\
\hline Na & & \\
\hline Hemoglobin & & \\
\hline eGFR & & \\
\hline
\end{tabular}

NYHA - New York Heart Association Functional Classification; LVEF - left ventricular ejection fraction; $\mathrm{Na}$ - sodium; eGFR - estimated glomerular filtration rate; ACE-I - angiotensin-converting enzyme inhibitors; hs-cTnT - high-sensitive cardiac troponin T; ST2 - suppression of tumorigenicity 2; NT-proBNP - N-terminal prohormone of brain natriuretic peptide.

is an independent prognostic value for all-cause mortality and hospitalization in patients with $\mathrm{CHF}^{43}$ Moreover, in a group of patients with dilated cardiomyopathy, sST2 correlated with all-cause mortality and combined outcome of death, left ventricle assist device (LVAD) implantation and cardiac transplantation. ${ }^{44}$

Moreover, significant interactions between sST2 level and HF treatment were suggested. In CHF patients treated with higher doses of $\beta$-blockers ( $>50 \mathrm{mg}$ of metoprolol succinate extended-release equivalent), sST2 concentrations dropped. ${ }^{45}$ Also, the higher the dose of $\beta$-blockers, the greater reduction in risk was found. ${ }^{45}$ Similar results were reported in the Valsartan HF Trial (Val-HeFT): a group treated with valsartan and a $\beta$-blocker had lower levels of sST2. ${ }^{46}$ Higher sST2 concentrations may indicate the increased risk of sudden cardiac death in patients with $\mathrm{HF}$ and reduced ejection fraction, ${ }^{47}$ which may help to identify the individuals who can benefit from an implantable cardioverter-defibrillator (ICD).

\section{Soluble ST2 and cardiosurgery}

Suppression of tumorigenicity 2 has the potential to be used in cardiac surgery. Patel et al. found that postoperative elevated plasma sST2 levels were associated with an increased incidence of cardiovascular event or mortality in adult patients who had undergone cardiac surgery. ${ }^{48}$ These findings were independent of pre-existing conditions such as CHF or acute kidney injury (AKI). Moreover, promising publications on the usefulness of sST2 in heart transplantation are appearing. Increased sST2 levels may be an indicator of acute allograft rejection in heart transplant recipients. ${ }^{49}$ It is suggested that the rejected graft is a source of serum sST2. Concentrations of sST2 are associated with an increased risk of antibody-mediated rejection after heart transplantation. ${ }^{50}$ This discovery opens the possibility for biopsy-free monitoring of anti-rejection therapy in heart transplant recipients. 


\section{Discussion}

Cardiac fibroblasts and cardiomyocytes produce circulating ST2 in response to stress and overload. ${ }^{12}$ The IL-33/ST2L axis has a cardioprotective effect and may, in the future, help create targeted therapy. ${ }^{35}$ Soluble ST2 is a biomarker which has the potential to be used for diagnosis and treatment monitoring in patients with various cardiovascular diseases. It is proving to be an especially important biomarker for both acute and chronic HF. ${ }^{35,36,40}$ Clinical data show promising results for the possible use of sST2 in various diseases, such as arrhythmia, hypertension, myocarditis, acute aortic syndrome, and CAD. ${ }^{30,28,22}$ This novel biomarker may play a role in heart transplantation and perioperative care of patients after cardiac surgery. This paper reviewed the literature on the possible applications of measuring SST2 in cardiology, where CAD and atherosclerosis are just one area of interest to researchers. In our opinion, the greatest interest in new biomarkers is currently focused on the area of HF. Heart failure is a significant and growing medical problem. The search for new biomarkers in this area is the opportunity to construct new hypotheses for HF pathogenesis and the opportunity to develop new therapies. The largest subsection is devoted to this problem. The ACC/AHA guidelines demonstrate the importance of this biomarker designation. ${ }^{34}$

\section{Conclusions}

Soluble ST2 is a promising biomarker in cardiology, especially in HF. However, further studies of the usefulness of sST2 in particular cardiac diseases are needed.

\section{ORCID iDs}

Magdalena Dudek (1) https://orcid.org/0000-0001-6550-6182 Marta Kałużna-Oleksy (D) https://orcid.org/0000-0003-4048-6247 Jacek Migaj (ㄴ) https://orcid.org/0000-0002-7962-3934 Ewa Straburzyńska-Migaj @ https://orcid.org/0000-0002-0545-3370

\section{References}

1. Biomarkers and surrogate endpoints: Preferred definitions and conceptual framework. Clin Pharmacol Ther. 2001;69(3):89-95. doi:10. 1067/mcp.2001.113989

2. Tominaga $S$. A putative protein of a growth specific CDNA from BALB/ $c-3 T 3$ cells is highly similar to the extracellular portion of mouse interleukin 1 receptor. FEBS Lett. 1989;258(2):301-304. doi:10.1016/00145793(89)81679-5

3. Schmitz J, Owyang A, Oldham E, et al. IL-33, an interleukin-1-like cytokine that signals via the IL-1 receptor-related protein ST2 and induces Thelper type 2-associated cytokines. Immunity. 2005;23(5): 479-490. doi:10.1016/j.immuni.2005.09.015

4. Tominaga S, Kuroiwa K, Tago K, I wahana H, Yanagisawa K, Komatsu N. Presence and expression of a novel variant form of ST2 gene product in human leukemic cell line UT-7/GM. Biochem Biophys Res Commun. 1999;264(1):14-18. doi:10.1006/bbrc.1999.1469

5. Iwahana $\mathrm{H}$, Hayakawa $\mathrm{M}$, Kuroiwa $\mathrm{K}$, et al. Molecular cloning of the chicken ST2 gene and a novel variant form of the ST2 gene product, ST2LV. Biochim Biophys Acta. 2004;1681(1):1-14. doi:10.1016/j.bbaexp. 2004.08.013

6. Ciccone MM, Cortese F, Gesualdo M, etal. A novel cardiac bio-marker:ST2. A review. Molecules. 2013;18(12):15314-15328. doi:10.3390/molecules 181215314
7. Mirchandani AS, Salmond RJ, Liew FY. Interleukin-33 and the function of innate lymphoid cells. Trends Immunol. 2012;33(8):389-396. doi:10.1016/j.it.2012.04.005

8. Liew FY, Pitman NI, Mclnnes IB. Disease-associated functions of IL-33: The new kid in the IL-1 family. Nat Rev Immunol. 2010;10(2):103-110. doi:10.1038/nri2692

9. Li R, Yang G, Yang R, Peng X, Li J. Interleukin-33 and receptor ST2 as indicators in patients with asthma: A meta-analysis. Int J Clin Exp Med. 2015;8(9):14935-14943.

10. Shi LJ, Liu C, Li JH, Zhu XY, Li YN, Li JT. Elevated levels of soluble ST2 were associated with rheumatoid arthritis disease activity and ameliorated inflammation in synovial fibroblasts. Chin Med J (Engl). 2018;131(3):316-322. doi:10.4103/0366-6999.223847

11. Boga S, Alkim H, Koksal AR, et al. Serum ST2 in inflammatory bowel disease: A potential biomarker for disease activity. J Investig Med. 2016;64(5):1016-1024. doi:10.1136/jim-2016-000062

12. Weinberg EO, Shimpo M, De Keulenaer GW, et al. Expression and regulation of ST2, an interleukin-1 receptor family member, in cardiomyocytes and myocardial infarction. Circulation. 2002;106(23): 2961-2966. doi:10.1161/01.cir.0000038705.69871.d9

13. Bartunek J, Delrue L, Van Durme F, et al. Nonmyocardial production of ST2 protein in human hypertrophy and failure is related to diastolic load. J Am Coll Cardiol. 2008;52(25):2166-2174. doi:10.1016/j. jacc.2008.09.027

14. Kakkar R, Lee RT. The IL-33/ST2 pathway: Therapeutic target and novel biomarker. NatRev Drug Discov. 2008;7(10):827-840. doi:10.1038/nrd2660

15. Shimpo M, Morrow DA, Weinberg EO, et al. Serum levels of the interleukin-1 receptor family member ST2 predict mortality and clinical outcome in acute myocardial infarction. Circulation. 2004;109(18): 2186-2190. doi:10.1161/01.CIR.0000127958.21003.5A

16. Hartopo AB, Sukmasari I, Puspitawati I. The utility of point of care test for soluble ST2 in predicting adverse cardiac events during acute care of ST-segment elevation myocardial infarction. Cardiol Res Pract. 2018;2018:1-9. doi:10.1155/2018/3048941

17. O'Donoghue ML, Morrow DA, Cannon CP, et al. Multimarker risk stratification in patients with acute myocardial infarction. J Am Heart Assoc. 2016;5(5):e002586. doi:10.1161/JAHA.115.002586

18. Somuncu MU, Kalayci B, Avci A, et al. Predicting long-term cardiovascular outcomes of patients with acute myocardial infarction using soluble ST2. Horm Mol Biol Clin Investig. 2020;41(2):/j/hmbci.2020.41. issue-2/hmbci-2019-0062/hmbci-2019-0062.xml. doi:10.1515/hmbci2019-0062

19. Bière L, Garcia G, Guillou S, et al. ST2 as a predictor of late ventricular remodeling after myocardial infarction. Int J Cardiol. 2018;259:40-42. doi:10.1016/j.ijcard.2018.02.058

20. Liu X, HuY, Huang W, et al. Soluble ST2 for prediction of clinical outcomes in patients with ST-segment elevation myocardial infarction receiving primary PCl. Int Heart J. 2019;60(1):19-26. doi:10.1536/ihj.18-020

21. Dieplinger B, Egger $M$, Haltmayer M, et al. Increased soluble ST2 predicts long-term mortality in patients with stable coronary artery disease: Results from the Ludwigshafen Risk and Cardiovascular Health Study. Clin Chem. 2014;60(3):530-540. doi:10.1373/clinchem.2013.209858

22. Zhang Y, Fan Z, Liu H, et al. Correlation of plasma soluble suppression of tumorigenicity-2 level with the severity and stability of coronary atherosclerosis. Coron Artery Dis. 2020;31(7):628-635. doi:10.1097/ MCA.0000000000000851

23. Wang $Y$, Tan X, Gao H, et al. Magnitude of soluble ST2 as a novel biomarker for acute aortic dissection. Circulation. 2018;137(3):259-269. doi:10.1161/CIRCULATIONAHA.117.030469

24. Morello F, Bartalucci A, Bironzo M, et al. Prospective diagnostic accuracy study of plasma soluble ST2 for diagnosis of acute aortic syndromes. Sci Rep. 2020;10:3103. doi:10.1038/s41598-020-59884-6

25. Coronado MJ, Bruno KA, Blauwet LA, et al. Elevated sera SST2 is associated with heart failure in men $\leq 50$ years old with myocarditis. J Am Heart Assoc. 2019;8(2):e008968. doi:10.1161/JAHA.118.008968

26. Coglianese EE, Larson MG, Vasan RS, et al. Distribution and clinical correlates of the interleukin receptor family member soluble ST2 in the Framingham Heart Study. Clin Chem. 2012;58(12):1673-1681. doi:10.1373/clinchem.2012.192153

27. Ojji DB, Opie LH, Lecour S, Lacerda L, Adeyemi OM, Sliwa K. The effect of left ventricular remodeling on soluble ST2 in a cohort of hypertensive subjects. J Hum Hypertens. 2014;28(7):432-437. doi:10.1038/ jhh.2013.130 
28. Farcas AD, Anton FP, Goidescu CM, et al. Serum soluble ST2 and diastolic dysfunction in hypertensive patients. Dis Markers. 2017;2017: 2714095. doi:10.1155/2017/2714095

29. Chen C, Qu X, Gao Z, et al. Soluble ST2 in patients with nonvalvular atrial fibrillation and prediction of heart failure. Int Heart J. 2018;59(1): 58-63. doi:10.1536/ihj.16-520

30. Nortamo S, Ukkola O, Lepojärvi S, et al. Association of sST2 and hsCRP levels with new-onset atrial fibrillation in coronary artery disease. Int J Cardiol. 2017;248:173-178. doi:10.1016/j.ijcard.2017.07.022

31. Okar S, Kaypakli O, Şahin DY, Koç M. Fibrosis marker soluble ST2 predicts atrial fibrillation recurrence after cryoballoon catheter ablation of nonvalvular paroxysmal atrial fibrillation. Korean Circ J. 2018;48(10): 920-929. doi:10.4070/kcj.2018.0047

32. Ibrahim NE, Lyass A, Gaggin HK, et al. Predicting new-onset HF in patients undergoing coronary or peripheral angiography: Results from the Catheter Sampled Blood Archive in Cardiovascular Diseases (CASABLANCA) study. ESC Heart Fail. 2018;5(3):240-248. doi:10.1002/ ehf2.12268

33. Mueller T, Jaffe AS. Soluble ST2: Analytical considerations. Am J Cardiol. 2015;115(7 Suppl):8B-21B. doi:10.1016/j.amjcard.2015.01.035

34. Yancy CW, Jessup M, Bozkurt B, et al. 2017 ACC/AHA/HFSA Focused update of the 2013 ACCF/AHA Guideline for the Management of Heart Failure: A report of the American College of Cardiology/American Heart Association Task Force on Clinical Practice Guidelines and the Heart Failure Society of America. J Am Coll Cardiol. 2017;70(6):776-803. doi:10.1016/j.jacc.2017.04.025

35. Januzzi JL, Peacock WF, Maisel AS, et al. Measurement of the interleukin family member ST2 in patients with acute dyspnea: Results from the PRIDE (Pro-Brain Natriuretic Peptide Investigation of Dyspnea in the Emergency Department) study. J Am Coll Cardiol. 2007;50(7): 607-613. doi:10.1016/j.jacc.2007.05.014

36. Mueller T, Dieplinger B, Gegenhuber A, Poelz W, Pacher R, Haltmayer M. Increased plasma concentrations of soluble ST2 are predictive for 1-year mortality in patients with acute destabilized heart failure. Clin Chem. 2008;54(4):752-756. doi:10.1373/clinchem.2007.096560

37. Boisot S, Beede J, Isakson S, et al. Serial sampling of ST2 predicts 90-day mortality following destabilized heart failure. J Card Fail. 2008;14(9):732-738. doi:10.1016/j.cardfail.2008.06.415

38. Demissei BG, Cotter G, Prescott MF, et al. A multimarker multi-time point-based risk stratification strategy in acute heart failure: Results from the RELAX-AHF trial. Eur J Heart Fail. 2017;19(8):1001-1010. doi: 10.1002/ejhf.749
39. van Vark LC, Lesman-Leegte I, Baart SJ, et al; TRIUMPH Investigators. Prognostic value of serial ST2 measurements in patients with acute heart failure. J Am Coll Cardiol. 2017;70(19):2378-2388. doi:10.1016/j. jacc.2017.09.026

40. Pascual-Figal DA, Ordoñez-Llanos J, Tornel PL, et al; MUSIC Investigators. Soluble ST2 for predicting sudden cardiac death in patients with chronic heart failure and left ventricular systolic dysfunction. J Am Coll Cardiol. 2009;54(23):2174-2179. doi:10.1016/j.jacc.2009.07.041

41. Bayes-Genis A, Zhang Y, Ky B. ST2 and patient prognosis in chronic heart failure. Am J Cardiol. 2015;115(7 Suppl):64B-69B. doi:10.1016/j. amjcard.2015.01.043

42. Lupón J, de Antonio M, Vila J, et al. Development of a novel heart failure risk tool: The Barcelona Bio-Heart Failure Risk Calculator (BCN bio-HF Calculator). PLoS One. 2014;9(1):e85466. doi:10.1371/journal. pone.0085466

43. Emdin M, Aimo A, Vergaro G, et al. sST2 predicts outcome in chronic heart failure beyond NT-proBNP and high-sensitivity troponin T. J Am Coll Cardiol. 2018;72(19):2309-2320. doi:10.1016/j.jacc.2018.08.2165

44. Wojciechowska C, Romuk E, Nowalany-Kozielska E, Jacheć W. Serum galectin-3 and ST 2 as predictors of unfavorable outcome in stable dilated cardiomyopathy patients. Hellenic J Cardiol. 2017;58(5):350-359. doi:10.1016/j.hjc.2017.03.006

45. Gaggin HK, Motiwala S, Bhardwaj A, Parks KA, Januzzi JL Jr. Soluble concentrations of the interleukin receptor family member ST2 and $\beta$-blocker therapy in chronic heart failure. Circ Heart Fail. 2013;6(6): 1206-1213. doi:10.1161/CIRCHEARTFAILURE.113.000457

46. Anand IS, Rector TS, Kuskowski M, Snider J, Cohn JN. Prognostic value of soluble ST2 in the Valsartan Heart Failure Trial. Circ Heart Fail. 2014;7(3):418-426. doi:10.1161/CIRCHEARTFAILURE.113.001036

47. Pascual-Figal DA, Pérez-Martínez MT, Asensio-Lopez MC, et al. Pulmonary production of soluble ST2 in heart failure. Circ Heart Fail. 2018;11(12):e005488. doi:10.1161/CIRCHEARTFAILURE.118.005488

48. Patel DM, Thiessen-Philbrook H, Brown JR, et al. Association of plasma-soluble ST 2 and galectin-3 with cardiovascular events and mortality following cardiac surgery. Am Heart J. 2020;220:253-263. doi:10. 1016/j.ahj.2019.11.014

49. Mathews LR, Lott JM, Isse $K$, et al. Elevated ST2 distinguishes incidences of pediatric heart and small bowel transplant rejection. Am J Transplant. 2016;16(3):938-950. doi:10.1111/ajt.13542

50. Grupper A, Abou Ezzeddine OF, Maleszewski JJ, et al. Elevated ST2 levels are associated with antibody-mediated rejection in heart transplant recipients. Clin Transplant. 2018;32(9):e13349. doi:10.1111/ctr.13349 